Horizons philosophiques

\title{
Instructions pour lire des photos de philosophes : Wittgenstein et la photographie
}

\section{Roch Duval}

Volume 11, numéro 1, automne 2000

L’amodernité de la photographie?

URI : https://id.erudit.org/iderudit/802949ar

DOI : https://doi.org/10.7202/802949ar

Aller au sommaire du numéro

Éditeur(s)

Collège Édouard-Montpetit

ISSN

1181-9227 (imprimé)

1920-2954 (numérique)

Découvrir la revue

Citer cet article

Duval, R. (2000). Instructions pour lire des photos de philosophes : Wittgenstein et la photographie. Horizons philosophiques, 11(1), 35-52.

https://doi.org/10.7202/802949ar d'utilisation que vous pouvez consulter en ligne.

https://apropos.erudit.org/fr/usagers/politique-dutilisation/ 


\title{
INSTRUCTIONS POUR LIRE DES PHOTOS DE PHILOSOPHES : WITTGENSTEIN ET LA PHOTOGRAPHIE.
}

\author{
La placa niega toda afirmación, \\ no hay evidencia alguna, sólo \\ conjeturas, rastros. \\ (...) \\ Imagen ciega sin su tradutor \\ marco de puerta en medio del desierto. \\ ¿Qué \\ - fuera de un asedio de imágenes - \\ no(s) dice esta placa?
}

Washington Benavides ${ }^{1}$

Lorsque je promène nonchalamment mon regard dans la pièce où habituellement je travaille, que ce soit en quête d'inspiration ou bien simplement pour apaiser un excès d'excitation causé par une imagination débridée, il m'arrive fréquemment de fixer l'une des nombreuses photographies de philosophes tapissant les murs de ce bureau, espérant secrètement y déceler un sourire approbateur, un clin d'œil complice, voire tout autre signe d'encouragement qui malheureusement - dois-je l'avouer - ne viennent jamais. Malgré le manque patent de collaboration de ces glorieux confrères ${ }^{2}$ - lesquels demeurent obstinément impassibles, imperturbables, pétrifiés derrière la vitre polie de leur cadre comme momifiés dans des "sarcophages

1. "La plaque photographique refuse toute affirmation/ il n'existe aucune évidence/ rien que des conjectures, des pistes/ (...)/ Image aveugle sans son traducteur/ encadrement de porte au milieu du désert/ Que (ne) nous dit (pas)/ - en dehors d'être un réceptacle d'images - cette plaque?"

Washington Benavides, Fotos (1983-1984), Montevideo, Ediciónes de la Banda Oriental, Poetas uruguayos de hoy \#11, 1986. L'ensemble des traductions, sauf indication contraire, sont les miennes.

2. Wolfgang Wackernagel joue sur l'étymologie des termes imago et amigo afin de mettre à nu une parenté philologique entre les termes Bild et $\phi i \lambda o s$. Cf. Wolfgang Wackernagel, "Subimaginale Versenkung. Meister Eckarts Ethik der Bild-ergrūnden Entbildung", dans Gottfried Boehm (éd.), Was ist ein Bild? (Qu'est-ce qu'une image?), Munich, Wilhelm Fink Verlag, 1994, p. 184-207. 
de l'interprétation ${ }^{3}$ " -, la contemplation de photos de philosophes, au-delà du pur plaisir esthétique qu'elles me procurent, a toujours exercé sur moi, malgré tout, un effet que je pourrais qualifier de roboratif. En effet, je dois candidement confesser que je collectionne depuis maintenant une quinzaine d'années des photographies ou (le cas échéant) des reproductions de peintures, représentant des philosophes illustres ou moins connus. Ainsi, dans le coin gauche de mon bureau, le Polonais Casimir Twardowski, au port altier, semble converser, contre toute attente, avec le très britannique George Frederik Stout. Dans l'autre coin, épuisés et éreintés par une vie dominée par une succession ininterrompue de débats houleux les opposant les uns aux autres, Karl Popper, Wilhelm Stegmüller, Paul Feyerabend et Thomas Kuhn se tiennent finalement cois. Avoisinant ce quatuor d'épistémologues, une sagaie africaine - offerte par le grand pacificateur qu'était le Père Lévesque - rappelle symboliquement les lances qu'ils ont rompues. À l'écart, faisant figure de cavalier seul, le malingre John Austin paraît engagé dans un soliloque sans fin. Une reproduction d'une peinture de René Descartes, de la main du peintre néerlandais Frans Hals (1585-1666), trône au-dessus d'un portrait d'Emmanuel Kant. Ces deux dernières images sont accrochées à proximité de mes diplômes; comme si le voisinage de ces grands maîtres avait pu communiquer, par une quelconque mystérieuse osmose ${ }^{4}$, une partie de leur inestimable savoir à mon humble condition!

De l'autre côté de la pièce, un mur, sur lequel se dresse fièrement une bibliothèque chargée de livres dont les différentes couvertures dessinent une mosaïque bigarrée, semble défier son vis-à-vis, où règne le monde de l'image. C'est dans cette atmosphère digne des meilleures photos de Clegg et Gutmann, où s'affrontent les mondes antagoniques de l'image et de l'écrit5, que scintille l'écran de mon ordinateur, commémorant ainsi à la fois la mort de la Galaxie

3. J'emprunte cette expression à Thomas Zaunschirm, "Vom Leben der Fotos" (De la vie des photos) dans Norbert Bolz et Ulrich Rüffer (éd.), Das große stille Bild (La photo grand format), Munich, Wilhelm Frank Verlag, 1996, p. 213-231, p. 221.

4. Vilém Flusser note avec justesse, quoique dans un sens différent, que le monde des images est un monde magique. Voir Vilém Flusser, Pour une philosophie de la photographie, traduit de l'allemand par Jean Mouchard, Circé, 1996, p. 10-11.

5. Cf. Vilém Flusser, op. cit., p. 7; Hans-Georg Gadamer, “Bildkunst und Wortkunst" dans Gottfried Boehm, op. cit., p. 90-104. 
Gutenberg et célébrant le retour en force de l'iconicité. Comme le souligne clairement Gernot Böhme :

La fin de la Galaxie Gutenberg, soutiennent certains observateurs, signifie non seulement la victoire des médias électroniques sur le monde de l'imprimé, mais également celle des images sur l'écriture6.

Le monde des images, singulièrement malmené depuis le début de l'ère moderne ${ }^{7}$, semble en passe de prendre sa revanche avec l'avènement des nouvelles technologies de l'information nous situant davantage dans une ère que nous pourrions qualifier de postmoderne8. D'aucuns ont même parlé d'un “ikonische Wendung" (Iconic Turn) ${ }^{9}$. Qu'en est-il alors de la photographie - elle-même

6. Theorie des Bildes (Théorie de l'image), Munich, Wilhelm Fink Verlag, 1999, p. 7.

7. Cf. Norbert Bolz, "Das große stille Bild im Medienverbund» (La photographie dans l'alliance des médias), dans Bolz et Rüffer, op. cit., p. 16-41.

Notons toutefois qu'au début de la modernité il a existé des "poches de résistance" militant contre la disparition du monde des images. Comenius, auteur du Orbis sensualium pictus (1658), constitue l'exemple le plus probant de cette rémanence du monde des images.

Au début du vingtième siècle, Otto Neurath, a également développé une méthode - dite ISOTYPE - dans laquelle l'iconicité joue un rôle primordial. Cf. Karl Heinz Müller, Symbol, Statistik, Computer Design. Otto Neuraths Bildpãdagogik im Computerzeitalter (Symbole, statistique, design des ordinateurs. La pédagogie des images d'Otto Neurath à l'ère des ordinateurs), Vienne, Hölder-Pichler-Tempsky, 1991. Voir également Andreas Roser, «Gibt es autonome Bilder? Bemerkungen zum grafischen Werk Otto Neurath und Ludwig Wittgenstein" (Y-a-t-il des images autonomes? Remarques sur l'oeuvre graphique d'Otto Neurath et Ludwig Wittgenstein), Grazer philosophische Studien, Vol. 52, (1996/97), p. 9-44.

8. Mark Poster dresse un constat saisissant des changements induits par le monde des nouvelles technologies. Voir en particulier The Second Media Age, Cambridge, Blackwell, 1995. Voir également l'entretien accordé par Poster à Eivind Røssaak, "Etter elektrifisiringen av spráket - samtale med Mark Poster" (Après l'électrification du langage - entretien avec Mark Poster) dans Eivind Røssaak, Det postmodeme og de intellektuelle. Essays og samtaler. (Le postmoderne et les intellectuels. Essais et entretiens), Oslo, Spartacus Forlag AS, 1998, p. 88-105.

9. Voir Gottfried Boehm, "Die Wiederkehr der Bilder" (Le retour des images), dans G. Boehm (éd.), op. cit., p. 11-38, p. 13; Oliver R. Scholz, Bild, Darstellung, Zeichen. Philosophische Theorien bildhafter Darstellung (Image, représentation, signe. Théories de la représentation picturale), Fribourg/Munich, Verlag Karl Alber, 1992; Gottfried Kerscher, alst der Iconic Turn auch für das Netz bzW. das WWW gültig?" (Le toumant iconique s'applique-t-il également à la Toile ou plutôt au réseau informatique international?), au site :

http:// www.rz.uni.frankfurt.de/ kerscher/nSem/ihih.html. 
produit paradigmatique de la modernité 10 - dans cet univers en constante mutation technique? L'ère de la photographie digitale signifie-t-elle irrémédiablement la disparition de la photographie classique?"11 Si l'on en croit Horst Wackerbarth'12, on ne doit pas s'inquiéter outre mesure du sort réservé à la photographie pour les années à venir; celle-ci s'appropriera du rôle que joue présentement la peinture et saura trouver une fonction à sa juste mesure dans l'univers du cyberespace ${ }^{13}$. C'est précisément par l'embrasure de la porte ouverte par l'avènement de la "Galaxie Turing"14 - supplantant la "Galaxie Gutenberg»15 - que s'engouffrent les images dans l'univers de la philosophie.

10. "(...) photography is modernity run riot", Allan Sekula, "The Body and the Archive", October 39, (1986), p. 4; La photographie est la première forme artistique à naître avec la société industrielle. Walter Benjamin note d'ailleurs que les débuts de la photographie coinncident avec l'émergence du socialisme. Cf. Walter Benjamin, "L'oeuvre d'art à l'époque de sa reproductibilité technique", dans Walter Benjamin, Sur l'art et la photographie, Présentation et traduction de Christophe Jouanlanne, Poitiers, Art \& Esthétique, 1997, p. 17-68, p. 30. Voir également Rolf $\mathrm{H}$. Kraus, Walter Benjamin und der neue Blick auf die Photographie, (Walter Benjamin et le nouveau regard sur la photographie), Stuttgart, 1998.

11. Voir Miklós Lehmann, “A digitális kép» (L'image digitale), au site :

http://193.225.239.10/ lehmann/digtkep.htm.

Le jeune philosophe hongrois Miklós Lehmann (né en 1968) a le mérite d'être également un photographe chevronné. Voir également, Lev Manovich, "Die Paradoxien der digitalen Fotografie" (Les paradoxes de la photographie digitale) dans Florian Rötzer (éd.), Fotographie nach der Fotographie (La photographie après la photographie), Dresde/Bâle, Verlag der Kunst, 1995.

Bernard Stiegler, quant à lui, troque le terme "photographie digitale" pour celui de "photographie analogo-numérique". Voir Bernard Stiegler, "Rémanence et discrétion des images", dans Art/Photographie numérique. L'image réinventée, Aix-en-Provence, CYPRES, École d'Art d'Aix-en-Provence, 1995, p. 220-252.

12. Horst Wackerbarth, "Die Ikonen des 21. Jahrhundrets" (Les icônes du $21^{\circ}$ siècle), dans Bolz/Rüffer, op. cit., p. 193-197.

13. À preuve l'achat par la société Corbis - une filiale de Microsoft - d'archives de photographies provenant d'agences spécialisées (des banques d'images) ou de photographes indépendants.

14. Voir Wolfgang Coy, «Bauelemente der Turingschen Galaxis" (Éléments constitutifs de la Galaxie Turing) dans Bulmahn, E., van Haaren, K., Hensche, D., Kieper, M., Kubicek, H., Rilling, R., Schmiede, R. (dir.), Informationsgesellschaft-Medien-Demokratie, (Société de l'information-MédiasDémocratie) Reihe Forum Wissenschaft, Marburg, Verlag BdWi, 1996.

15. Cf. Norbert Bolz, Am Ende der Gutenberg Galaxis : die neuen Kommunikationsverhältnisse, (Au bout de la Galaxie Gutenberg: les nouveaux rapports de communication), Munich, Wilhelm Fink Verlag, 1993. 
Paraphrasant le poète uruguayen Rafael Courtoisie, nous pourrions dire que l'entreprise poursuivie dans ce texte consiste cette fois-ci à donner des instructions non pas pour lire des cendres mais plutôt des photographies ${ }^{16}$. Si l'image des cendres peut symboliquement évoquer la perte du savoir résultant de l'incendie de la Bibliothèque d'Alexandrie ${ }^{17}$, la soi-disant mort des livres proclamée par l'avènement de la Galaxie Turing ne laisse pour sa part aucune cendre ${ }^{18}$. Le passage de la littéralité à l'univers de l'iconicité/oralité19 une sorte d'Aufhebung - et par la suite le va-et-vient incessant entre ces deux univers (intermédia) ${ }^{20}$, moyennant un certain effort interprétatif, implique une persistance du contenu informatif. II s'agit en quelque sorte d' "abluer" symboliquement les plaques photographiques afin que la signification jusque là celée puisse pleinement se révéler21.

Tirant parti d'un projet mené par Timoty Dow Adams 22 visant à établir une convergence entre la pratique de l'autobiographie et celle de la photographie, j'aspire à démontrer comment on peut interpréter la philosophie de Wittgenstein en exposant ce qu'il dit sur la photographie et comment, en retour, la photographie peut révéler quelque

16. Rafael Courtoisie, Instrucciónes para leer ceniza (Cambio de estado) (Instructions pour lire des cendres (Changement d'état)), Montevideo, 1990.

17. «Entre las páginas perdidas de Alejandría se quemó ésta». Rafael Courtoise, op. cit.

18. Dietmar Kamper (1999) dit : "Les ordinateurs furent construits par des sapeurspompiers, non par des pyromanes". Voir Dietmar Kamper, Ein Cursor. In sieben Stücken, (Un curseur. En sept fragments), au site:

http//www.digitab.de/kamp/cursor/cursor2.htm.

19. Ou plus précisément à ce que Walter Ong nomme l' "oralité du second ordre". À ce propos, voir la pénétrante analyse du philosophe Kristóf Nyíri, "Bevezetés : Adalékok a szóbeliség-írásbeliség paradigma történetéhez" (“Introduction : Sur l'histoire du paradigme oralité-littéralité"), dans K. Nyíri et G. Szécsi, (éd.), Szóbeliség és írásbeliség : A kommunikációs technológiák története Homérosztól Heideggerig (Littéralité et oralité. Histoire des technologies de la communication de Homère à Heidegger), Budapest, Áron Kiadó, 1998, p.7-17.

20. Voir Peter Gärdenfors, "Intermedia" dans Ibid., Det fangslande information (L'information captive), Stockholm, Natur och Kultur, 1996, p. 36-45.

21. Contrairement aux cendres qui effacent les messages inscrits sur le papier ou sur tout autre support, les photographies ravivent - en les présentant sous un nouvel angle - les symboles qui apparaissent à leur surface. Comme le dit si bien l'écrivain espagnol Ramón Goméz de la Serna, maître incontesté des "gregerías" - cette philosophie des instantanés - "La única que guarda un secreto es la ceniza". (La cendre est la seule qui sait garder un secret).

22. Voir Timoty Dow Adams, Light Writing \& Life Writing. Photography in Autobiography, Chapel Hill, The University of North Carolina Press, 2000. 
chose sur lui. Bien qu'une théorie de la photographie soit absente chez ce dernier, il semble bien que l'on puisse tout de même déduire des "instructions" servant à l'interprétation des photographies. "Every instruction can be construed as a description, every description as an instruction ${ }^{23}$ ". Mon intention consiste donc à donner une description de ce que Wittgenstein dit sur la photographie ${ }^{24}$. L'objectif visé ne concerne donc pas l'élaboration d'une théorie wittgensteinienne de la photographie mais uniquement la formulation d'cinstructions" (description) servant à interpréter des photos. Pour mener à bien cette étude, j'utilise tant les écrits de la période de transition, la correspondance et le journal intime de Ludwig Wittgenstein ${ }^{25}$ qu'une certaine photographie de ce dernier ${ }^{26}$.

\title{
Wittgenstein et la photographie
}

\author{
Der findes ingen \\ virkelighed, \\ vi samler den selv: \\ Billeder, billeder

\section{Naja Marie Aid27}

Une des caractéristiques de la philosophie de Wittgenstein concerne la place qu'il accorde tout au long de sa carrière au monde des images. Andreas Roser, s'appuyant lui-même sur les travaux de M. Biggs, répertorie 1300 illustrations (schémas, diagrammes,

23. Ludwig Wittgenstein, Philosophical Remarks 14, Chicago, The University of Chicago Press, p. 59.

24. Pour bien comprendre la démarche poursuivie dans ce texte, il s'agit de remplacer "langage" par "photographie" dans cette citation de Wittgenstein : al am only describing language, not explaining anything". Philosophical Grammar 30, p. 66.

25. Denkbewegungen, Tagebücher 1930-1932, 1936-1937, Haymon-Verlag, Dr. Michael Forscher, Innsbruck, 1997. J'utilise, pour ma part, la version norvégienne de ce journal intime. Ludwig Wittgenstein. Den ukjente dagboken. Cambridge 1930-32 og Skjolden 1936-37. Traduction et introduction de Knut Olav Ámás, épilogue de Jon Fosse, Oslo, Spartacus Forlag AS, 1998. À partir de maintenant cet ouvrage sera désigné par les lettres UD.

26. La photographie en question est reproduite, entre autre, dans Michael Nedo et Michele Ranchetti (éd.) : Wittgenstein, Sein Leben in Bildern und Texten. Francfort, Suhrkamp, 1983.

27. "ll n'existe aucune réalité/nous l'assemblons nous-même : Images, images", Naja Marie Aidt,

Det tredje landskab (Le troisième paysage), Copenhague, Gyldendal, 1994, p. 41. 
dessins, etc.) dans les textes de Wittgenstein ${ }^{28}$. À cela s'ajoute une quantité non moins impressionnante de photographies du philosophe autrichien. Prenant à témoin ces deux sources, je tente de rassembler dans ce qui suit les maigres observations de Wittgenstein sur la photographie et d'en tirer, si possible, quelques observations pertinentes.

D'entrée de jeu une remarque préliminaire s'impose. Bien que la première philosophie de Wittgenstein - celle du Tractatus (1921) - fait de la notion de Bild le socle à partir duquel il érige sa conception du langage comme image ou tableau logique du monde (Bildtheorie der Sprache), on ne saurait commettre l'erreur visant à confondre "image" (Bild) et photographie (Lichtbild) ${ }^{29}$. Bien que le terme allemand "Lichtbild" - une des nombreuses manières d'écrire le terme photographie (Aufnahme, Lichtbild, Photo, Foto) - contienne comme élément le mot "Bild»30, les deux termes renvoient à des choses totalement différentes. Notons premièrement que le terme Bild désigne indistinctement une multitude de choses - image, illustration, figure, tableau, portrait, effigie, idole et parfois même photographie ${ }^{31}$-, alors que le terme Lichtbild désigne uniquement le mot photographie 32 . Cela étant dit, on ne doit pas confondre la Bildtheorie élaborée par Wittgenstein avec ses remarques éparses, incidentes et non formelles sur la photographie, bien que ses remarques générales sur les images - principalement dans ses écrits

28. Voir Michael Biggs, "A Source Catalogue of the Published Diagrams", dans Wittgenstein : Two Sources Catalogues and a Bibliography, M. Biggs et A. Pichler (éd.), Bergen, 1993 (cité dans Roser, op. cit., p. 10).

29. Cf. Thomas Hölscher, "Bild" und "Grammatik" bei Wittgenstein." ("ulmage» et "grammaire" chez Wittgenstein) dans Klaus-Sachs Hombach et Klaus Rehkämper (éd.), Bildgrammatik (Grammaire de l'image), Magdeburg, Scriptum Verlag, 1999, p. 69-78. Voir également de Miklós Lehmann, "Wittgenstein korai és kései képfelfogásáról” (Sur la théorie des images dans la philosophie première et tardive de Wittgenstein) au site :

http://www.phil-inst.hu/-lehmann/wkepek.htm.

30. Sur cet aspect, la langue allemande ressemble au hongrois et au finnois qui forment le terme photographie à partir des mots "image" et "lumière" (fénykép : fény (lumière) et kép (image) pour le hongrois; valokuva : valo (lumière) et kuva (image) pour le finnois).

31. Voir G. Böhme, op. cit., p. 7.

32. Picasso était lui-même conscient de la grande différence qui existe entre le monde des images créées intentionnellement par l'homme (Bild) et celui des images produites mécaniquement ou artificiellement (photographies). Les photos ne sont pas des tableaux (Bild) car elles apparaissent uà une plus grande vitesse que les images». Pablo Picasso, Écrits, Paris, Gallimard, 1989, p. 213. 
intermédiaires où il s'affaire à reformuler la Bildtheorie - puissent se révéler utiles. Nous prenons le parti de nous limiter exclusivement à ses observations sur la photographie.

Un incident survenu à Øystese (Norvège, octobre 1913), impliquant David Hume "Pinsent et Ludwig Wittgenstein, peut servir d'introduction au thème qui nous intéresse. Pinsent avait sa caméra avec lui et il délaissa Wittgenstein un instant afin de prendre une photo. Lorsqu'il rejoignit son compagnon de voyage, ce dernier était silencieux et boudeur. Aucun mot ne fut échangé pendant une demi-heure. Pinsent voulut savoir ce qui n'allait pas. II s'avéra que Wittgenstein était très irrité par l'ardeur qu'avait mis son ami à prendre des photos. II s'était comporté comme un homme qui ne peut penser à rien d'autre, lorsqu'il se promène en plein air, que comment un paysage peut se transformer en un terrain de golf» 33 .

Que penser de cet incident? La colère de Wittgenstein était-elle dirigée contre l'amateurisme de Pinsent ou bien contre la photographie en tant que pratique? Notons que le manque de considération de Wittgenstein envers les photographes amateurs (comme David Hume Pinsent) ne peut guère être généralisé à la photographie en général. Une des (rares) amies de Wittgenstein - fortement estimée par lui de surcroît - était elle-même une photographe professionnelle. Lettice Cautley Ramsey (1898-1985), épouse du non moins célèbre Frank Plumpton Ramsey, se révéla être une photographe de renom à l'emploi de la firme Ramsey and Muspratt, Cambridge photographers, et Wittgenstein prenait plaisir à contempler ses épreuves. II semble donc que l'incident d'Oystese doit être perçu non pas comme une critique de la photographie en général mais bien comme une réaction (excessive?) du philosophe viennois envers son compagnon de voyage. La question demeure à savoir dans quelle mesure Wittgenstein estimait véritablement la photographie.

Notons d'entrée de jeu qu'on ne saurait facilement dénicher dans les écrits de Wittgenstein une appréciation esthétique de la photographie comparable à l'affection qu'il vouait au cinéma. Sa passion pour le cinéma est bien connue. Le 6 mai 1930 il notait dans son journal intime :

33. Knut Olav Ámás et Rolf Larsen, Det stille alvoret. Ludwig Wittgenstein i Norge 1913-1950. Etterord av Kjartan Flogstad (La tranquillité sereine. Ludwig Wittgenstein en Norvège 1913-1950. Épilogue de Kjartan Fløgstad), Oslo, Det Norske Samlaget, 1994, p. 81. 


\begin{abstract}
Dans un sens je dois être un homme très moderne, puisque le cinéma exerce sur moi un effet immensément bénéfique. Je ne peux m'imaginer plus heureux que visionnant un film américain. (UD, p. 38. Je souligne)
\end{abstract}

Cette remarque de Wittgenstein peut surprendre si l'on considère que, somme toute, il était un individu très conservateur, voire rétrograde, et que ses valeurs étaient fortement ancrées dans les traditions de l'univers austro-hongrois, depuis peu moribond 34 . Comment accueillir ce soi-disant umodernisme» de Wittgenstein? Bien qu'il fût au courant des conceptions théoriques d'Adolf Loos sur l'architecture (9 mai 1930, UD, p. 41), on ne peut présumer qu'il connaissait également les travaux théoriques afférents aux autres courants esthétiques ou idéologiques émergeant à cette époque. La méconnaissance des réflexions théoriques et philosophiques sur la photographie de Lázsló Moholy-Nagy, Siegfried Kracauer ou bien Raoul Hausmann, de concert avec son ignorance de la réflexion philosophique sur le cinéma de Béla Balasz, s'expliquent vraisemblablement en raison du fait que Wittgenstein ne désirait pas encombrer son esprit de conceptions étrangères aux siennes. Dans une lettre à von Wright, il dévoile cette particularité de sa personnalité : "(...) in order to live and to work, I have to allow no import of foreign goods (i.e. philosophical ones) into my mind 35 ". Or selon une interprétation véhiculée par le philosophe hongrois Kristóf Nyíri, l'apparition des premiers films parlant (“talkies») aurait exercé un effet non négligeable dans la formation de l'ultime philosophie du viennois ${ }^{36}$. Les premiers films parlant firent leur apparition en 1928 à Londres puis en 1929 à Vienne. Or c'est précisément vers la fin de 1929 et le début des années 30 que l'on note les premières observations de Wittgenstein sur la photographie. II est important de saisir que cette période coïncide avec la période de remise en

34.Voir les observations de Kristóf Nyíri quant au conservatisme politique et culturel de Wittgenstein dans A hagyomány filozófiája (La philosophie de la tradition). Budapest, T-Twins- Lukács Archivum, 1994.

35. Lettre du 21 février 1947, Letters from Ludwig Wittgenstein to Georg Henrik von Wright, dans James Klagge et Alfred Nordmann (dir.), Ludwig Wittgenstein. Philosophical Occasions 1912-1951, Indianapolis, Hackett, 1993, p. 462.

36. Kristóf Nyíri, "Wittgenstein videon : Kényszer - találkozás Jarman filmjével» (Wittgenstein sur vidéo : Rencontre forcée avec le film de Jarman), Metropolis 1997/4, p. 104-110. Voir en particulier la section intitulée "Wittgenstein és a mozi" (Wittgenstein et le cinéma). Voir également du même auteur "Wittgenstein as a Philosopher of Second Orality" dans Grazer Philosophische Studien, Vol. 52 (1996/97), p. 45-58. 
question de la Bildtheorie du Tractatus. La première mention des photographies remonte précisément au 27 novembre 1929 c'est-à-dire à l'occasion de la conférence qu'il prononça à Cambridge pour la Heretics Society. En effet, dans "A Lecture on Ethics", Wittgenstein introduit la méthode de la photographie composite de Francis Galton afin d'expliquer la nature de l'éthique :

And to make you see as clearly as possible what I take to be the subject matter of Ethics I will put before you a number of more or less synonymous expressions each of which could be substituted for the above definition, and by enumerating them I want to produce the same sort of effect which Galton produced when he took a number of photos of different faces in the same photographic plate in order to get the picture of the typical features they all had in common. And as by showing to you such a collective photo I could make you see what is the typicalæsay Chinese face; so if you look through the row of synonyms which I will put before you, you will, I hope, be able to see the characteristic features they all have in common and these are the characteristics features of Ethics. ${ }^{37}$

L'approche galtonienne a indubitablement influencé Wittgenstein car il en fait mention à maintes reprises ${ }^{38}$. Lasynchronie entre l'introduction de la procédure des photos composites dans la panoplie des exemples de Wittgenstein et les linéaments de la formulation de la théorie des jeux de langage a de quoi surprendre. On peut vraisemblablement avancer l'hypothèse que la pratique photographique de Galton a servi de modèle à l'élaboration de cette théorie. Wittgenstein n'offre cependant aucune preuve quant à une telle influence et cette hypothèse demeure à être corroborée.

Bien que selon la conception courante les photographies soient supposées représenter la réalité telle qu'elle est - car elle en serait une émanation, une trace ${ }^{39}$-, on sait pertinemment que cette opinion est erronée ${ }^{40}$. La photographie peut présenter des objets usuels,

37. Ludwig Wittgenstein, "A Lecture on Ethics" dans ibid., Philosophical Occasions 1912-1951, James Klagge et Alfred Nordmann (éd.), Indianapolis, Hackett, 1993, p. 37-44, p. 38. On peut comparer ce passage à celui de la Grammaire philosophique 36.

38. Philosophical Remarks (1930), p. 293; The Blue Book (1933-34) p. 18; "The Language of Sense Data and Private experience" (1936), p. 305; Wiener Ausgabe 5-170-37.

39. Voir Herbert Hrachovec, “Fotogene Enttäuschungen» (Désillusions photogéniques) dans Deutsche Zeitschrift für Philosophie 43 (1995), p 455-463. 
communs, connus, dans une perspective qui ne nous est pas familière. La photographie est dotée de cette double capacité de modifier la perspective spatiale (agrandissement, vue à vol d'oiseau, vue panoramique) et la dimension temporelle (photos de mouvements décomposés de Muybridge) d'objets communs ${ }^{41}$. L'interprétation des photos doit être soumise au même traitement auquel on soumet les mots, les images ordinaires ou le langage. Tout comme la signification d'un mot est révélée par l'usage que l'on en fait dans un énoncé, une photographie doit s'interpréter selon les éléments qui lui sont co-constitutifs. On doit apprendre à lire (à décoder) les photographies non pas en les comparant à la réalité 42 mais en respectant leur "grammaire".

Let us remember too that we don't have to translate such pictures into realistic one in order to 'understand' them, any more than we ever translate photographs or film pictures into coloured pictures, although black-and-white men or plants in reality would strike us as unspeakably strange and frightful. (Zettel 242)

C'est vraisemblablement ce que voulait dire Wittgenstein le 6 mai 1931, lorsqu'il notait dans son journal :

La photographie de mon frère Rudi a quelque chose d'Oberländer ou plus précisément quelque chose du style des bons dessins dans les vieux Fliegende Blätter. (U.D. p. 63)

La photographie de son frère Rudolf (1881-1904), un mince petit carré noir et blanc, donne une image "caricaturale" d'où la mention du nom du caricaturiste Adolf Oberländer au service du journal humoristique Fliegende Blätter (1844-1944) - par rapport à une réalité (qui serait une sorte de noumène inaccessible et qui forme un concept incompatible avec l'anti-essentialisme de Wittgenstein) de celui-ci. La réalité véhiculée par la photo du petit Rudi est inhérente à la photo elle-même et réside dans l'interprétation qu'en donne Wittgenstein. Le réalisme d'une photographie ne réside pas dans une

40. Voir le dernier chapitre du livre de Gernot Böhme op. cit. «Ist ein Foto realistisch? " (Une photo est-elle réaliste?), p. 111-127.

41. Voir Peter Weibel, “Das fotografisches Großbild im Zeitalter der Geschwindigkeit" dans Boll/Rüffer, op. cit., p. 46-71.

42. "Welche Beziehung hat die Vorstellung von einer Photographie die ich heute gesehen habe zu der Photographie (dem Gesichtbild der Photographie). Ist sie Ihr ăhnlich? Vourausgezetz daß sie genau mit der Wirklichkeit übereinstimmt (was eben bei einer Vorstellung mit der Wirklichkeit" heißt)". Ludwig Wittgenstein, Wiener Ausgabe 3-102-(8-12). 
adéquation entre un original et son modèle mais doit être interprété selon la grammaire propre aux images. On ne trouve guère d'autres observations intéressantes de Wittgenstein sur la photographie. II reste maintenant à interpréter une photo du philosophe viennois afin d'apprendre quelque chose sur lui.

\section{Wittgenstein et son négatif}

Par conséquent, une photographie peut être considérée comme déchiffrée dès lors qu'on est parvenu à établir quelle est en elle la part de collaboration et quelle est la part de lutte.

\section{Vilém Flusser 43}

L'ultime étude que Roland Barthes consacra à la photographie 44 désarçonna la légion de lecteurs qui espéraient y trouver une théorie achevée de la photographie. Sans passer ici en revue les mérites et les démérites de la réflexion barthienne - car on ne saurait véritablement parler ici d'une théorie - metionnons qu'il y existe au moins un élément digne d'être rescapé. Cet élément est formé de la paire studium/punctum 45 . Sans tenter d'ériger en absolu cette dichotomie qui, soit dit en passant, n'en est pas une mais indique plutôt une complémentarité dans la lecture ou dans l'interprétation des photos -, je désire aborder la description d'une certaine photographie en prenant comme angle d'approche la notion de punctum ${ }^{46}$. Compulsant fébrilement ma pinacothèque/photothèque privée 47 , je constate qu'une certaine photographie me point plus que toutes les autres.

La photographie se présente comme suit. Deux hommes, tous deux assis dans des chaises de jardin, fixent l'objectif de la caméra. L'un d'eux présente un visage renfrogné, porte des sandales et une chemise sport. L'autre arbore une chemise blanche sur laquelle se découpe une cravate noire et affiche une mine réjouie. L'arrière-fond est blanc. On devine plus que l'on perçoit un tronc d'arbre, quelques

43. Pour une philosophie de la photographie, p. 51.

44. Roland Barthes, La chambre claire. Note sur la photographie, Paris, Gallimard/Seuil, 1980.

45. Barthes, ibid., p. 48-49.

46. "Le punctum d'une photo, c'est ce hasard qui, en elle, me point (mais aussi me meurtrit, me poigne)". Barthes, ibid., p. 49.

47. Sur la notion de pinacothèque privée voir de László Moholy-Nagy, "Peinture, photographie, film" dans ibid., Peinture, photographie et film et autres écrits sur la photographie, traduit de l'allemand par Catherine Wermester et de l'anglais par J. Kempf et G. Dallez. Préface de Dominique Baqué, Nîmes, Éditions Jacqueline Chambon, 1993, p. 89-91. 
brindilles, un peu de gazon ainsi que la base de ce qui semble être une marche.

En quoi cette photographie me point-elle? Quelle est son punctum? En quoi m'interpelle-t-elle? La photographie en question a ceci d'unique qu'elle met en scène non pas un philosophe mais bien deux philosophes : Ludwig Wittgenstein et son éventuel dauphin, Georg Henrik von Wright. C'est précisément dans cet état de fait (dual et duel) que réside le caractère spectaculaire de cette photographie. La représentation de deux philosophes sur une même photographie brise le carcan conceptuel auquel nous a habitués la (jeune) tradition photographique ${ }^{48}$. Nul besoin de mentionner qu'en règle générale les photographies de philosophes se ressemblent toutes : on y perçoit uniquement un buste se détachant d'un arrière-fond monochrome une sorte de version de la photo de passeport, pose hiératique qu'un code vient légiférer. Dès 1864, dans The Camera and the Pencil, le photographe américain Marcus Aurelius Root prescrit les règles à suivre : les individus vivant d'activités sédentaires (poètes, historiens, professeurs, philosophes) doivent être photographiés assis; la position debout convient aux hommes d'action ou influents (hommes d'état, ecclésiastiques, industriels). II existe ces photos, dites officielles, représentant les philosophes imbus d'eux-mêmes, l'air blasé et empesé, le sourire timide (lorsque celui-ci n'est pas carrément inexistant), le regard vide, le cou qu'étrangle une cravate sobre. On a beau scruter ces photographies à la recherche d'éléments révélateurs (verrue, rides, calvitie, monocle, chaîne de montre, boutons d'argent, collet amidonné, moustache, pince à cravate) mais rien ne parvient à dissiper cet air de déjà-vu. À preuve, l'étonnante ressemblance existant entre la photographie officielle de John Austin et celle du philosophe finlandais Erik Ahlmann : le même visage émacié, la même esquisse de sourire, les mêmes lunettes à la monture noire, la même coupe de cheveu, la même pose guindée, la même coupe de veston, la même cravate, etc. Parfois, des photographies que je pourrais qualifier de "circonstancielles" viennent rompre cette monotonie.

Je pense en particulier à certaines photos de Paul Feyerabend où l'on voit celui-ci, tablier cintré à la taille, en train de laver la vaisselle dans son appartement romain ou bien tentant d'imiter le King Kong du poster épinglé au mur de son bureau à Berkeley. Ou bien encore,

48. Cf. Leena Saraste, Valokuva tradition ja toden välissä (La photographie entre la tradition et la réalité), Helsinki, Musta Taide, 1996. 
cette photographie de Harlingue représentant Henri Bergson à sa table de travail. Considérant que la philosophie est perçue comme une activité solitaire 49 où domine la pugnacité, il n'est donc pas étonnant que les philosophes soient presque toujours représentés seuls. Les rares cas de photographies de philosophes où ceux-ci sont représentés en compagnie d'autres personnes sont des mises en scène où les philosophes apparaissent:

1) Avec leur famille. Par exemple :

Eino Kaila en compagnie d'Anna Snellman-Kaila, son épouse, et Gerty Wichmann se prélassant sur des rochers sur les rives d'un lac en Finlande en 1938;

Jalmari Edvard Salomaa assis dans le salon de sa demeure à Turku, entouré de sa femme, son fils et sa fille, en 1943;

Georg Henrik von Wright en compagnie de son épouse Elisabeth, tout deux revêtant des vêtements de gala, lors d'une cérémonie officielle tenue le 5 mai 1990 à Helsinki.

2) En groupe. Par exemple :

les 26 participants espagnols au congrès portant sur le fondement des droits humains à Madrid, le 20 avril 1988;

la photo de groupe du congrès sur l'épistémologie de la physique quantique à Singapour, en 1987.

3) Dans une foule. Ce type de photo met particulièrement en valeur des philosophes militants comme Jean-Paul Sartre, Michel Foucault ou Bernard Henri-Levy, défendant le plus souvent une cause sociale.

Il est utile de remarquer ici que l'environnement dépeint dans ces photos n'est jamais hostile. On peut certes noter une gradation dans l'ordre de l'impersonnalité (famille, groupe que cimente un intérêt commun, foule), mais aucun de ces environnements ne remet en cause le philosophe lui-même : sa figure demeure le point focal vers lequel converge le regard et les autres personnages deviennent des satellites gravitant autour de lui.

Comme nous l'avons dit, la photographie de Wittgenstein/von Wright constitue un événement unique. Le caractère duel de cette photo - un fait déjà remarquable en soi - ne doit pas laisser dans l'ombre, ou plutôt dans un angle mort, cet élément supplémentaire :

49. "The philosopher is not a citizen of any community of ideas. That is what makes him into a philosopher". Ludwig Wittgenstein, Zettel, Anscombe et von Wright (éd.), traduit par Anscombe, no 455, p. 81. 
cette photo duelle est en réalité une photographie mettant en scène un triplet de philosophes - Wittgenstein, von Wright et Knut Erik Tranøy (le photographe) : le Père, le Fils, le Saint Esprit!

Je vais tenter de (dé)montrer comment cette photographie est à la fois duelle/duale et que von Wright y apparaît comme le "négatif" de Wittgenstein, c'est-à-dire celui à partir de qui Wittgenstein peut se révéler.

II existe premièrement une opposition au niveau des couleurs. En effet, tandis que le philosophe viennois porte une chemise noire, le philosophe finlandais arbore une chemise blanche sur laquelle se découpe une cravate noire contrastant avec le maillot de corps blanc que l'on sent naître sous le collet de la chemise de Wittgenstein. Les cheveux pâles de Wittgenstein contrastent avec les cheveux foncés de von Wright. Ce contraste se poursuit si on examine cette fois-ci les chaussettes de ceux-ci. Les sandales de Ludwig laissent voir des chaussettes blanches, alors que l'on perçoit les chaussettes noires de Georg Henrik révélées par un repli du pantalon. La jambe gauche croise la jambe droite du maitre alors que la jambe droite croise la jambe gauche du disciple.

II existe ensuite une différence au niveau des attitudes. Pendant que l'auteur du Tractatus présente un visage renfrogné, le visage du futur créateur de la logique déontique est illuminé par un sourire complice. Une entrée dans le journal de Wittgenstein, en date du 9 octobre 1930, nous permet vraisemblablement de comprendre cette attitude peu amène. Wittgenstein écrit :

Même si je me retrouve parmi des gens aimables (ou bien peut-être est-ce pour cette raison?) je me sens tout le temps ennuyé - même si ceux-ci ne me dérangent pas physiquement - voilà pourquoi je peux difficilement me joindre à un groupe. C'est une situation horrible. Chaque mot que je les entends prononcer m'ennuie. (UD, p. 47)

La description amorcée ci-dessus serait incomplète si on ne glissait mot de l'arrière-plan de la photographie. Derrière Wittgenstein et von Wright un drap blanc fait écran, masquant l'entrée de la véranda de la maison du Finlandais. Se fiant à ce que narre Knut Erik Tranøy, il appert que l'idée de tendre un drap serait venue de Wittgenstein lui-même. De surcroît, ce dernier aurait insisté pour que le drap utilisé soit le sien et non pas un drap net provenant de l'armoire d'Elisabeth von Wright. Que déduire de cette mise en scène 
peu habituelle?50 Avançons l'hypothèse (l'allégorie) suivante : le drap tendu par Wittgenstein ferait autant office de lange que de linceul. L'insistance démontrée par Wittgenstein à utiliser son propre drap (souillé!?) exprime le désir d'accoucher éventuellement (et finalement) d'une œuvre. Quand invite-t-on un étranger à partager son drap si ce n'est pour engager une activité copulatoire, procréatrice? La lecture du journal intime de Wittgenstein laisse percevoir l'angoisse qui habitait celui-ci à l'idée de n'avoir rien laissé de valable derrière lui, de ne pouvoir passer à la postérité. L'image juxtaposée de Wittgenstein et von Wright couchés sur le drap de Wittgenstein doit être interprétée comme l' "intromission" de l'esprit (fragmenté/ désordonné/inachevé/larvaire) de Wittgenstein dans le corps (méthodique/rassembleur/synthétique) de von Wright. N'eut été l'œuvre éditoriale du Finlandais, aurions-nous pu parler de Wittgenstein de nos jours? II ne faut jamais oublier que le Wittgenstein que nous connaissons (par l'entremise de ses "écrits") est une pure création de von Wright (et des autres exécuteurs testamentaires ayant participé à l'élaboration de son œuvre). Le drap blanc symboliserait en quelque sorte le lange servant à emmailloter l'œuvre/rejeton à naître.

Cette interprétation s'avère cependant partielle car il s'agit également de (dé)montrer que le drap blanc peut également symboliser un linceul. Lorsque Tranøy immortalisa cette scène, vers la fin du printemps 1950, le philosophe viennois se savait déjà condamné par le mal incurable qui aurait finalement raison de lui. Cette conscience de l'imminence de la mort, ressentie comme la fin du processus de la création, pourrait également expliquer le regard cynique de Wittgenstein. Remarquez comment les véritables créateurs ne fixent jamais l'objectif de la caméra. Seuls les individus en-deçà ou en au-delà ou bien carrément exclus du processus de la création osent le faire. Susan Sontag avait déjà remarqué que seuls les politiciens ou les pathétiques modèles photographiés par Diane Arbus osaient regarder effrontément la caméra. 51 Comparez les photographies ornant les pochettes des disques de John Coltrane avant et pendant

50. "The conventional portrait photograph is never accidental, can never be something "taken" by chance; rather it is necessarily arranged, agreed upon : the subject must consciously consent to the occasion". Celia Lury, Prosthetic Culture. Photography, Memory and Identity, Routledge, Londres, 1998, p. 45.

51. Susan Sontag, Sur la photographie, traduction de Philippe Blanchard, Paris, Seuil, 1983. Le livre posthume de Diane Arbus, Untitled (1996), est entièrement consacré à la photographie de malades mentaux. 
la période créatrice. Les photos de Bob Cato et d'Esmond Edwards mettent en scène un jeune Coltrane avant l'explosion créatrice 52 : le saxophoniste fixe alors candidement l'objectif de la caméra. Les photos de Bob Thiele et de Charles Stewart montrent en revanche un autre Coltrane : celui déjà engagé dans le processus de la création 53 . Le regard se détourne, la paupière se ferme, l'ombre envahit le visage, l'éclairage trop vif efface les traits. Ou bien le créateur se présente résolument de profil comme dans la magnifique photo du microsillon $A$ Love Supreme. N'existe-t-il pas un mythe autour du visage actuel de Réjean Ducharme que seule une photographie surannée confirme l'existence physique? N'y-a-t-il pas eu jadis un branle-bas de combat orchestré par Alvaro Guillot-Muñoz afin de trouver une photo de Lautréamont ${ }^{54}$. Les créateurs fuient l'œil indiscret et impudique de la caméra. Ce n'est qu'arrivé au terme du processus créateur que l'artiste daigne présenter de nouveau son visage, dévoiler son regard; 55 cette fois-ci non plus en signe de provocation mais pour signifier son abdication face à la mort.

Une certaine tristesse, une résignation dans le regard, lie d'ailleurs la photographie de Wittgenstein à la troublante photo du poète danois Michael Strunge (1958-1986). Vidé de son génie créateur -11 recueils en 7 ans! -56 , Strunge fixe tristement la caméra sachant déjà que son heure est venue : le suicide sera son dernier poème. Tranøy rapporte qu'initialement Wittgenstein avait exprimé le désir d'être photographié de dos. Je ne sais guère ce qui a bien pu se passer dans la tête du Viennois pour que celui-ci se ravise et élabore la mise en scène constituant le décor de la photo précitée.

52. John Coltrane, Coltrane Time, United Artist, UAS 5638; John Coltrane, Coltrane, Prestige, OJC-020 (P-7105) (1957).

53. John Coltrane, A Love Supreme, Impulse A-77 (1964); John Coltrane Featuring Pharoah Sanders, Live in Seattle, Impulse 2027-9202 (1965).

54. Cf. Jacques Lefrère, Le visage de Lautréamont, Paris, Pierre Horay, 1977.

55. Cf. les observations de Wittgenstein sur le regard : "We do not see the human eye as a receiver, it appears not to let anything in, but to send something out. The ear receives; the eye looks. (It casts glances, it flashes, radiates, gleams.) One can terrify with one's eye, not with one's ear or nose. When you see the eye you see something going out from it. You see the look in the eyen, Zettel, \#222, p. 40.

56. Michael Strunge, Samlede Strunge. Digte 1978-1985 (L'oeuvre intégrale de Strunge. Poésie 1978-1985), Copenhague, Borgen, 1991. 


\title{
Conclusion
}

\author{
Consejo supertilosófico : "Hágase una fotografía y si \\ sale es que existen.

\section{Ramón Gómez de la Serna57}

À ce jour, il n'existe aucune étude portant sur le thème de la photographie chez Ludwig Wittgenstein. Nous avons tenté, dans un premier temps, de rassembler et de commenter quelques mentions que fait Wittgenstein de la photographie. Dans un second temps, j'ai formulé une hypothèse quant au rôle joué par la pratique des photographies composites de Galton dans l'élaboration de la notion des jeux de langage. Finalement, nous avons isolé une photographie du philosophe viennois afin de donner des instructions pour une lecture possible de celle-ci.

$\mathrm{Au}$ terme de ceci, des questions demeurent entières. Comment concilier le réalisme patent de la procédure galtonienne des photos composites à l'anti-réalisme (le nominalisme) de sa philosophie du langage? En quel sens sa perception de la photographie a-t-elle été instrumentale dans la remise en question de la Bildtheorie du Tractatus? En quoi la photographie contribue-t-elle à sa critique de la notion de ressemblance (Ähnlichkeit)58? Quelle est la relation entre une photographie et la notion de référence 59 ? En quoi le style littéraire de Wittgenstein ressemble-t-il au style photographique? Ces questions, et tant d'autres encore, sont encore à la recherche d'une réponse. Cet état de fait constitue un appel criant à la poursuite d'investigations ultérieures.

\section{Roch Duval Centre d'étude et d'interprétation de la pensée scandinave}

57. “Conseil superphilosophique : "Fais-en une photographie et si ça paraît c'est que ça existe"». Ramón Gómez de la Serna, Greguerias, Madrid, Edición de Antonio A. Gómez Yebra (Clasicos Castadia), 1994.

58. “Ein Mensch/Einer/dem man eine Photographie des A zeigt und - den man fragt findest Du nicht daß inm die Photographie sehr ăhnlich sieht kőnnte sagen. Keine Spur, sie ist gar nicht ähnlich : das Papier ist viereckig und ganz dünn und er nicht..." Ludwig Wittgenstein, Wiener Ausgabe 3-106-38. Voir également 3-132-44.

59. Ludwig Wittgenstein, "The Language of Sense Data and Private Experience", dans ibid., Philosophical Occasions, p. 328-29. 